\title{
Evaluación del Médico Interno de Pregrado a través de Competencias Profesionales en Medicina Familiar
}

\section{Assessment of the Undergraduate Intern trough Professional Competences in Family Medicine}

DOI: $10.46932 / \mathrm{sfjdv} 2 \mathrm{n} 4-016$

Received in: March 1st, 2021

Accepted in: May 30th, 2021

\section{Perla Patricia Abarca de Hoyos}

Médico residente de medicina familiar. Unidad de Medicina Familiar No. 80. Instituto Mexicano del Seguro Social.

\section{Gerardo Muñoz Cortés}

Doctor en Ciencias de la Educación. Coordinador Clínico de Educación e Investigación en Salud. Unidad de Medicina Familiar No. 80. Instituto Mexicano del Seguro Social. Av. Madero 1200, colonia centro, C.P. 58000, Morelia, Michoacán, México.

Correo electrónico: gerardomunozcortes@gmail.com

\section{María Dolores Martínez Calvo}

Médico residente de medicina familiar. Unidad de Medicina Familiar No. 80. Instituto Mexicano del Seguro Social.

\section{María Elena Estrada Andrade}

Doctora en Ciencias de la Educación. Universidad Michoacana de San Nicolás de Hidalgo

\section{Yurixhi Cancino Estrada}

Médico Familiar. Unidad de Medicina Familiar No. 75. Instituto Mexicano del Seguro Social.

\section{Laura Miriam Pérez Flores}

Médico Familiar. Profesora titular del curso de especialización en medicina familiar. Unidad de Medicina Familiar No. 80. Instituto Mexicano del Seguro Social.

\section{Carlos Gómez Alonso}

Matemático. Coordinador analista. Universidad Michoacana de San Nicolás de Hidalgo.

\section{RESUMEN}

Introducción: Los métodos de evaluación aplicados al área de la salud continúan siendo tradicionales, donde se evalúan conocimientos sin captar habilidades, actitudes y aptitudes. Es necesario buscar una estrategia para evaluar la competencia profesional en los médicos internos de pregrado (MIP) por medio de un examen clínico objetivo estructurado (ECOE)

Objetivo: Evaluar las competencias profesionales de los médicos internos de pregrado que egresan de su rotación por medicina familiar.

Material y métodos: Estudio cuasiexperimental, prospectivo, longitudinal realizado a los médicos internos de pregrado que cursaron medicina familiar en la Unidad de Medicina Familiar No. 80 en los bimestres: Enero-Febrero, Marzo-Abril y Julio-Agosto del 2020. Se llevó a cabo una evaluación diagnóstica y de acuerdo al resultado se estableció un programa académico y se realizó una evaluación posterior de las competencias profesionales, mediante un examen clínico objetivo estructurado. Se utilizó 
estadística descriptiva, para comparación de medias: $\mathrm{t}$ de student, significancia $\mathrm{p}<0.05$. Registro SIRELCIS R-201-1602-023.

Resultados: Se evaluaron 23 médicos internos de pregrado, $65.2 \%$ mujeres; con $23.34 \pm 0.48$ años. Nivel de competencia al inicio $21.7 \%$ y $100 \%$ al final de la evaluación. Media de conocimientos al iniciar 5.6 y al finalizar 5.8 (p 0.06). Excelencia en conocimientos $2.1 \%$, en habilidades $92.7 \%$; en actitud y aptitud $100 \%$ al finalizar la rotación.

Conclusión: Los médicos internos de pregrado son competentes al egresar de su rotación de medicina familiar.

Palabras clave: Competencias profesionales, médico interno, actitud, aptitud, habilidades, conocimientos.

\begin{abstract}
Introduction: The evaluation methods applied to the health areas continue to be traditional, written exams that assess knowledge without capturing skills, attitudes and aptitudes. It is necessary to seek a strategy to assess the professional competence of undergraduate medical interns through a objective structured clinical examination (OSCE).

Objective: Assess the professional competences of the undergraduate interns who complete their service in family medicine

Material and methods: Quasi-experimental, prospective, longitudinal study of undergraduate interns who are studying family medicine in the UMF 80 in the bimesters: January-February, March-April and July-August of 2020, was made. Diagnosis assessment was applied and with the result a bimonthly academic program was planned. A final evaluation of professional competences was carried out with the objective structured clinical examination. Descriptive statistics were used for comparison of means: $t$ of student, significance $\mathrm{p}<0.05$. SIRELCIS R-201-1602-023.

Results: We assessed 23 undergraduate interns, $65.2 \%$ women; with $23.34 \pm 0.48$ years. Competence level at the beginning of $21.7 \%$ and $100 \%$ at the end of the evaluation. Mean knowledge at the beginning of 5.6 and 6.8 at the end (p 0.06). Excellence in knowledge 2.1\%, in skills 92.7\%; in attitude and aptitude $100 \%$ at the end of the evaluation.

Conclusion: undergraduate medical interns are competent to graduate from their rotation of family medicine.
\end{abstract}

Keywords: Professional competences, intern, attitude, aptitude, skills, knowledge.

\title{
1 INTRODUCCIÓN
}

Los retos que ofrece una sociedad en constante proceso de cambio son de tal magnitud que se exige que los estudiantes, desarrollen sus capacidades generales junto con sus conocimientos y puedan encajarlos en el marco de las necesidades sociales del entorno (Beneitone, 2014).

Las competencias profesionales las define Rodríguez en 2007 como la integración de conocimientos, destrezas y actitudes que permiten el desempeño profesional de calidad. Son actuaciones integrales ante actividades y problemas del contexto, con idoneidad y compromiso ético, integrándose el saber ser, el saber hacer y el saber conocer en una perspectiva de mejora continua (Tobón, 2010). 
La educación basada en competencias es un reto importante en la sociedad que se construye en el siglo XXI, afirma Salas-Flores en 2016, donde la calificación profesional ya no es concebida únicamente como la acumulación de saberes o habilidades, sino como la capacidad de actuar, intervenir y decidir en situaciones no siempre previstas; así el foco de atención se ha desplazado de las calificaciones a las competencias profesionales. Ello conlleva a un proceso en el que las universidades se vinculan a la sociedad y específicamente en el área del trabajo, tratando de alcanzar la integridad formativa que permita al educando adquirir los conocimientos (saber), las habilidades (saber hacer), las aptitudes (poder hacer) y las actitudes (querer hacer) que garantice las competencias profesionales requeridas y lograr comportarse a la altura de su tiempo (saber ser) (Vidal, 2016).

Cresp (2018) afirma que para que los cambios en la enseñanza tengan real repercusión en el aprendizaje de los estudiantes, es preciso que existan transformaciones en las prácticas de evaluación.

La sociedad demanda un médico que se comunique de manera abierta y flexible con el paciente; colabore con sus pares y utilice la mejor evidencia para tomar decisiones, diagnosticar y resolver problemas de salud (Martínez-González, 2017).

Tapia (2007) comenta que el internado de pregrado es una etapa del plan de estudio de las facultades de medicina para la formación del médico general y se considera como un periodo obligatorio previo al servicio social para que los estudiantes integren y consoliden los conocimientos teóricos y prácticos adquiridos durante los ciclos escolarizados previos a la carrera. Durante esta etapa se espera que los alumnos se incorporen a los servicios de salud para que desarrollen nuevas competencias en el terreno de la atención integral de los problemas de salud (Sánchez, 2008). En este año los estudiantes tienen mayor responsabilidad con los pacientes y se pueden fortalecer sus habilidades y mejorar su desempeño clínico (Trejo, 2014). En su programa existen rotaciones por las principales ramas de la medicina como medicina familiar, medicina interna, pediatría, ginecología, urgencias y cirugía; donde permanecerán dos meses en cada servicio.

En la actualidad los internos de medicina están expuestos a una serie de problemas, como la sobrecarga laboral, deficiencias en la docencia, estrés, agotamiento físico y emocional, repercutiendo no solo en la calidad de vida y la salud mental, sino también en su rendimiento académico (Nieto-Gutiérrez, 2016).

Las competencias en Medicina las definió Soler en 2004 como conocimientos, habilidades y actitudes caracterizados en modos de actuación, aplicados en la solución de los problemas de salud.

La evaluación de la competencia clínica se ha realizado a través del tiempo utilizando instrumentos como el examen oral y el examen ante paciente real; pero el avance de la psicometría ha permitido determinar la baja confiabilidad de estas herramientas, las cuales han sido desplazadas por el examen 
clínico objetivo estructurado (ECOE), que se considera actualmente el método con mayor grado de objetividad para evaluarla (Trejo-Mejía, 2013).

Para Martínez (2018) el ECOE consiste en una serie de estaciones por las que el alumno pasa en donde se plantean situaciones clínicas simuladas para resolver y en este se valoran dos dimensiones: la cognitiva (conocimientos y la psicomotriz/actitudinal (habilidades/actitudes/aptitudes). Para su implementación se requiere personal docente con capacitación específica en la creación de escenarios y respectivos guiones, cuyos objetivos deben estar alineados con los objetivos generales del curso y malla curricular; permitiendo otorgar un feedback inmediato al alumno de parte del evaluador (Ticse, 2017).

El objetivo de esta investigación fue lograr evaluar las competencias profesionales a los médicos internos de pregrado que egresan de su rotación por medicina familiar del Instituto Mexicano del Seguro Social e implementar esta forma de evaluación para generaciones futuras, ya que es un método que actualmente no se ha aplicado en el Instituto y con esto se logrará egresar estudiantes que sean capaces de integrar todo lo que un exige un perfil profesional.

\section{MATERIAL Y MÉTODOS}

Estudio cuasiexperimental, prospectivo, longitudinal que se realizó a 23 médicos internos de pregrado adscritos al Hospital General Regional No. 1 del Instituto Mexicano del Seguro Social durante el ciclo 2019-2020 y 2020-2021. Para el cálculo del tamaño de la muestra se utilizó la fórmula de población finita. Se incluyeron los médicos internos de pregrado que se encontraban rotando por medicina familiar en la Unidad de Medicina Familiar No. 80 en los bimestres Enero-Febrero del 2020, Marzo-Abril del 2020, Julio-Agosto del 2020, independientemente de la edad y sexo. No se incluyeron los médicos internos de pregrado que se incapacitaran durante más del $20 \%$ de asistencia y los que no aceptaron participar en el estudio. Se excluyeron los que no cumplieron con el $80 \%$ de las asistencias o los que no aceptaron continuar con el protocolo.

Previa autorización del protocolo por parte del comité local de ética en investigación en salud (R2019-1602-023), se llevó a cabo una reunión inicial con los médicos internos de pregrado de cada bimestre previamente mencionado y se les invitó a participar en el estudio.

Aquellos que firmaron un consentimiento informado y aceptaron participar en el estudio: Se les realizó una evaluación diagnóstica que consistió en la aplicación de un examen basado en las asignaturas del programa académico de los médicos internos de pregrado durante su rotación por medicina familiar.

La evaluación diagnóstica inicial de las competencias que incluyó la aptitud, la actitud y habilidad para atender pacientes, se evaluó con el examen clínico objetivo estructurado, el cual se realizó en un consultorio de medicina familiar establecido en la Unidad de Medicina Familiar No. 80 (UMF 80) como 
consultorio escuela, donde se adaptaron 6 estaciones y en éstos se incluyeron casos clínicos, análisis de estudios, estudios de gabinete y presencia de pacientes simulados representando las principales patologías de nuestro medio: diabetes mellitus, hipertensión arterial, sobrepeso y obesidad, control prenatal, infecciones de vías urinarias, infecciones vaginales; donde el alumno mostró necesidades de capacitación. Apoyados por médicos pasantes del servicio social adscritos a la UMF 80 que actuaron como pacientes, con un guión previamente establecido y estudiado.

Se evaluó mediante aplicación de listas de cotejo por parte de la médico residente de medicina familiar de acuerdo a cada patología, para estimar las competencias profesionales de los médicos internos.

Para evaluar la actitud, independientemente de la patología evaluada, se categorizó en "excelente" cuando realizaron 6 acciones (saludar, presentarse con el paciente y acompañante, invitarlo a sentarse, ser receptivo, empático y respetuoso); "bueno” cuando realizó 4 o 5 acciones; "regular” cuando realizó 3 acciones y "deficiente" cuando realizó 2 o menos acciones.

Para evaluar la aptitud, independientemente de la patología evaluada, se categorizó en "excelente" cuando realizaron las 5 acciones (detectar signos, detectas síntomas de la patología, selección e interpretación de exámenes de laboratorio, selección de estudios de laboratorio, selección de estudios de gabinete y efectuar acciones preventivas encaminadas a conservar o preservar la salud); "bueno" cuando realizaron 4 acciones; "regular" cuando realizaron 2 o 3 acciones y "deficiente" cuando realizaron 1 o ninguna acción.

Para evaluar habilidades se realizó una lista de cotejo para cada una de las patologías:

Para habilidades en diabetes mellitus: se categorizó en "excelente" cuando realizaron 4 acciones ( solicitar > 2 estudios correspondientes: glucosa sérica, hemoglobina glucosilada y curva de tolerancia oral a la glucosa; comprender y analizar los resultados; solicitar estudios de función renal, calcular la tasa de filtración glomerular y estadificar correctamente el daño renal; tomar $>6$ puntos de exploración de pie diabético y realizar agudeza visual); "bueno" cuando realizaban 3 acciones; "regular" cuando realizaban 2 acciones y "deficiente” si solo realizaban 1 o ninguna acción.

Para habilidades en hipertensión arterial: se categorizó en "excelente" cuando realizaron 4 acciones (solicitar electrocardiograma; calcular el índice de Sokolow-Lyon e interpretarlo; solicitar radiografía de tórax y calcular el índice cardiotorácico); "bueno" cuando realizaban 3 acciones; "regular" cuando realizaban 2 acciones y “deficiente” si solo realizaban 1 o ninguna acción.

Para habilidades en obesidad: se categorizó en "excelente" cuando realizaron 4 acciones (pesar y medir al paciente; calcular adecuadamente el índice masa corporal, interpretar el resultado y medir el perímetro abdominal); "bueno" cuando realizaban 3 acciones; "regular" cuando realizaban 2 acciones y “deficiente" si solo realizaban 1 o ninguna acción. 
Para habilidades en control prenatal: se categorizó en "excelente" cuando realizaron 4 acciones (calcula adecuadamente la edad gestacional, solicita e interpreta estudios de laboratorio y gabinete, explica e interpreta datos de alarma); "bueno" cuando realizaban 3 acciones; "regular" cuando realizaban 2 acciones y "deficiente” si solo realizaban 1 o ninguna acción.

Para habilidades en infecciones de vías urinarias: se categorizó en "excelente" cuando realizaron 4 acciones (identificar síntomas urinarios, solicitar examen general de orina, interpretarlo adecuadamente y otorgar datos de alarma); "bueno" cuando realizaban 3 acciones; "regular" cuando realizaban 2 acciones y "deficiente" si solo realizaban 1 o ninguna acción.

Para habilidades en infecciones vaginales: se categorizó en "excelente" cuando realizaron 4 acciones (interrogar sobre sintomatología de infección vaginal, interpretar características del flujo vaginal, solicitar cultivo vaginal e interpretarlo adecuadamente); "bueno" cuando realizaban 3 acciones; "regular" cuando realizaban 2 acciones y "deficiente” si solo realizaban 1 o ninguna acción.

La evaluación diagnóstica del nivel de conocimientos se realizó a través de un. examen de conocimientos con respuestas de opción múltiple, que incluyó reactivos de las 6 patologías, fueron realizados en el aula 1 de la UMF 80 y cuyos reactivos fueron calibrados mediante el método Rasch.

De acuerdo a los resultados se creó un programa académico enfocados en las debilidades y posteriormente se realizó una intervención con clases teóricas y prácticas por medio de clases presenciales en el aula 1 de la UMF 80, 3 días a la semana, 1 hora diaria, cubriendo un total de 24 horas clase y se procedió a realizar una evaluación final de la aptitud, actitud, habilidades y conocimientos para comparar la competencia profesional y los conocimientos después de la intervención. Se obtuvieron datos de todas las evaluaciones y se realizó el registro en el instrumento de recolección por alumno para su análisis posterior.

Para el análisis estadístico los resultados se presentaron en medias \pm desviación estándar para las variables continuas, mientras que las variables categóricas se presentaron en porcentajes. Comparación de medias a través de una t de student para muestras relacionadas. Todos los cálculos se realizaron con el paquete estadístico SPSS versión 23.0 para Windows. Se consideró con significancia estadística a un valor de $\mathrm{p}<0.05$ y se midió la fiabilidad de los instrumentos de evaluación mediante alfa de cronbach. El instrumento de evaluación objetiva se calibró mediante el modelo Rasch, a través del programa ministep.

\section{RESULTADOS}

Se incluyeron 23 médicos internos de pregrado que cumplieron los criterios de selección. A continuación se muestran las características generales de los participantes, nótese el predominio de mujeres, egresados de la Universidad Michoacana de San Nicolás de Hidalgo. Tabla I. 


\begin{tabular}{|l|c|}
\hline \multicolumn{2}{|c|}{ Tabla I. Características de los médicos internos de pregrado (n=23) } \\
\hline & $\mathbf{X} \pm \mathbf{D E}$ \\
\hline Edad (años) & $23.34 \pm 0.48$ \\
\hline Sexo & $\mathbf{F ~ ( \% )}$ \\
\hline Hombre & $8(34.8 \%)$ \\
\hline Mujer & $15(65.2 \%)$ \\
\hline Universidad de egreso & $22(95.6 \%)$ \\
\hline Universidad Michoacana de San Nicolás de Hidalgo & $1(4.3 \%)$ \\
\hline Universidad Autónoma de Guadalajara & \\
\hline
\end{tabular}

$\mathrm{X}=$ Promedio. $\mathrm{DE}=$ Desviación estándar. $\mathrm{F}=$ Frecuencia.

Se evaluaron las seis patologías más comúnmente tratadas en la consulta de primer contacto, en seguida se muestran los resultados de las calificaciones obtenidas por los médicos internos al iniciar y finalizar la rotación por medicina familiar, observándose el incremento mayor en sobrepeso, infecciones vaginales y urinarias. Tabla II.

\begin{tabular}{|l|c|c|}
\hline \multicolumn{1}{|c|}{ Tabla II. Calificación inicial y final de los médicos internos de pregrado } \\
en los 6 temas prioritarios (n=23)
\end{tabular}

* t de student muestras pareadas, p 0.06 (Significancia $\mathrm{p}<0.05)$

En la figura 1 se observa que el nivel de conocimientos de la mayoría de los médicos internos de pregrado iniciaron en la categoría de deficiente y finalizaron en categoría de regular. 
Figura 1: Nivel de conocimientos antes y después de la intervención educativa.

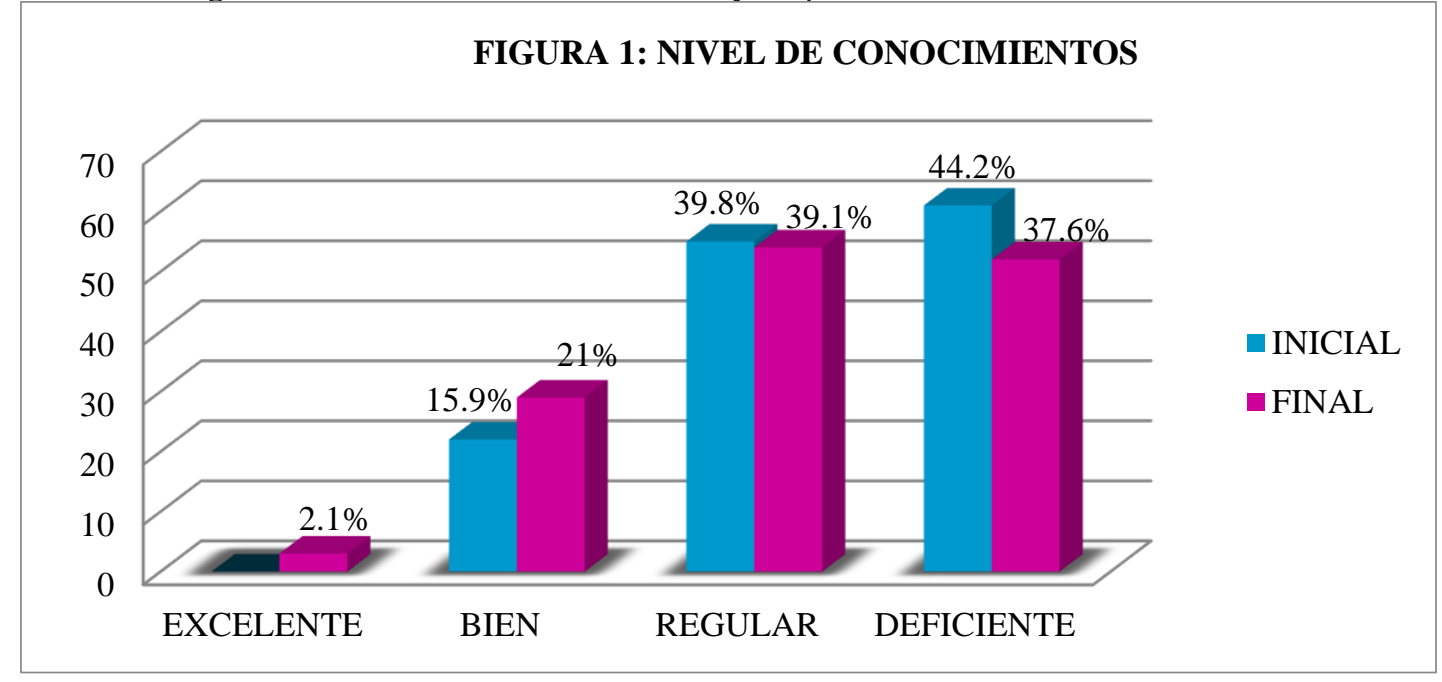

Se evaluaron las habilidades de los médicos internos ante cada patología, donde se calificó de acuerdo a los niveles de ejecución para cada atributo. Nótese el crecimiento en las seis patologías. Ver tabla III.

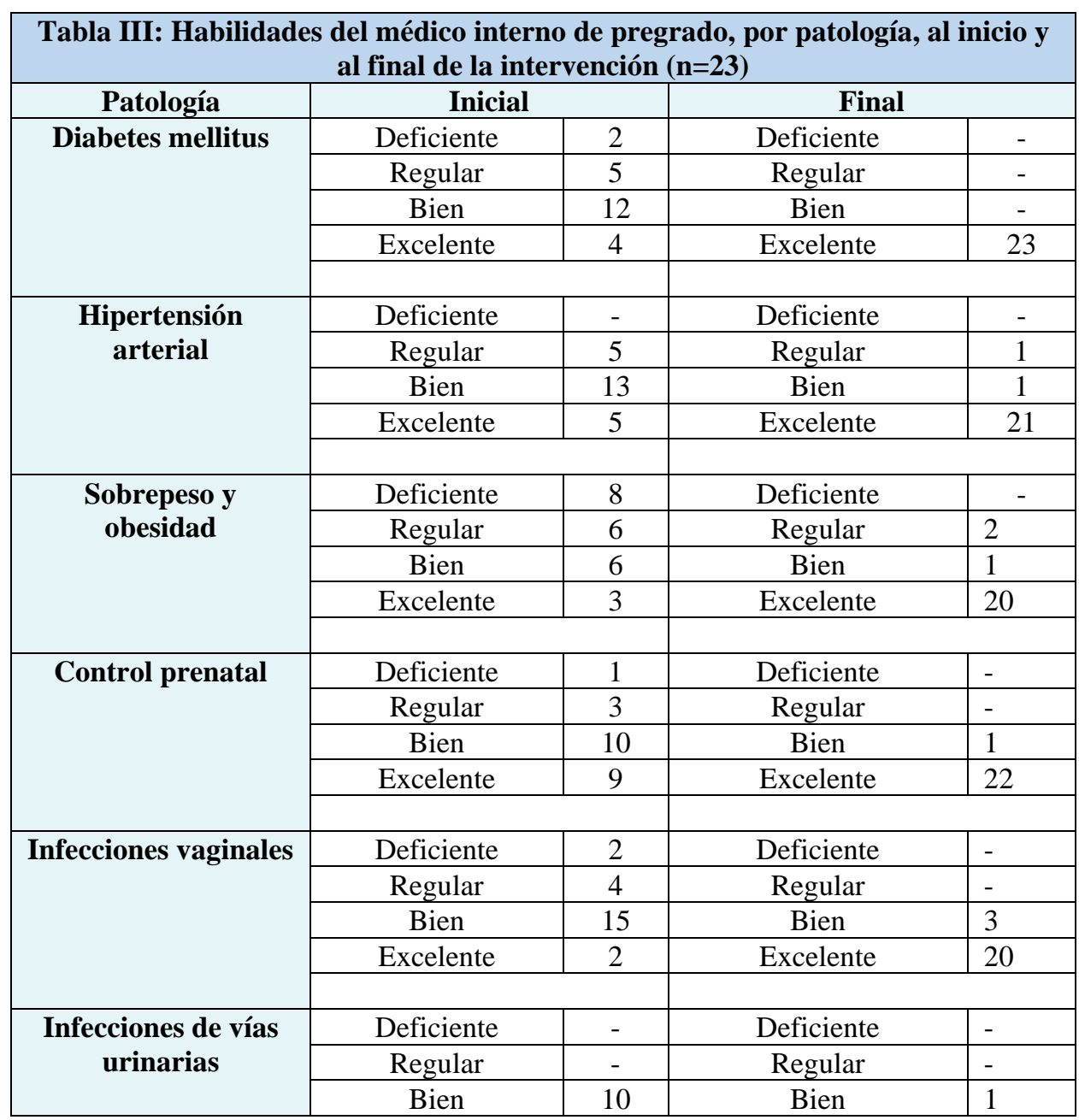




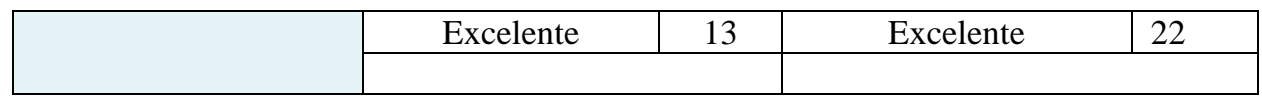

En la figura 2 se muestra que la mayoría de los médicos internos de pregrado finalizaron su rotación por medicina familiar con habilidades excelentes, posterior a la intervención educativa que se realizó.

Figura 2. Habilidades de los Médicos internos de pregrado antes y después de la intervención educativa.

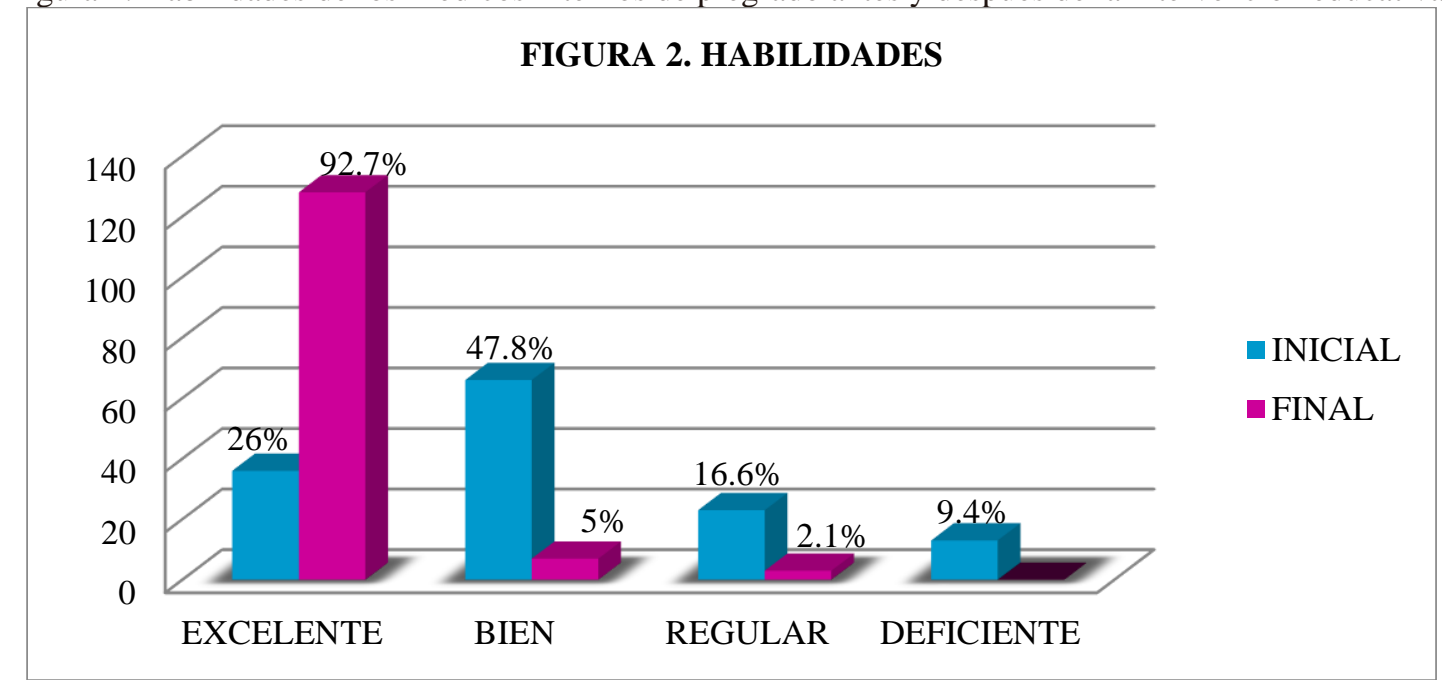

Dentro de las competencias también se evaluaron la actitud y aptitud de los médicos internos; en la tabla IV se observa el desarrollo que presentaron en la evaluación final colocándose la mayoría de los alumnos en área de excelencia, excepto por una alumna que culminó en la categoría de bien.

\begin{tabular}{|c|c|c|c|c|c|c|c|c|}
\hline & \multicolumn{8}{|c|}{$\begin{array}{l}\text { Tabla IV: Aptitud y Actitud del médico interno de pregrado al } \\
\text { inicio y al final de la intervención }(n=23)\end{array}$} \\
\hline & \multicolumn{4}{|c|}{ INICIAL } & \multicolumn{4}{|c|}{ FINAL } \\
\hline & Calific & & $\mathbf{H}$ & $\mathbf{M}$ & Calific & & $\mathbf{H}$ & $\mathbf{M}$ \\
\hline \multirow[t]{4}{*}{ Aptitud } & Deficiente & - & - & - & Deficiente & - & - & - \\
\hline & Regular & 2 & - & 2 & Regular & - & - & - \\
\hline & Bien & 15 & 5 & 10 & Bien & 1 & - & 1 \\
\hline & Excelente & 6 & 3 & 3 & Excelente & 22 & 8 & 14 \\
\hline \multirow[t]{4}{*}{ Actitud } & Deficiente & - & - & - & Deficiente & - & - & - \\
\hline & Regular & - & - & - & Regular & - & - & - \\
\hline & Bien & 7 & 2 & 5 & Bien & - & - & - \\
\hline & Excelente & 16 & 6 & 10 & Excelente & 23 & 8 & 15 \\
\hline
\end{tabular}


En la figura 3 se observa la actitud de los médicos internos de pregrado al iniciar y finalizar su rotación por medicina familiar, donde observamos que el total de los alumnos culminaron con una excelente actitud después de la intervención educativa.

Figura 3. Actitud antes y después de la intervención educativa.

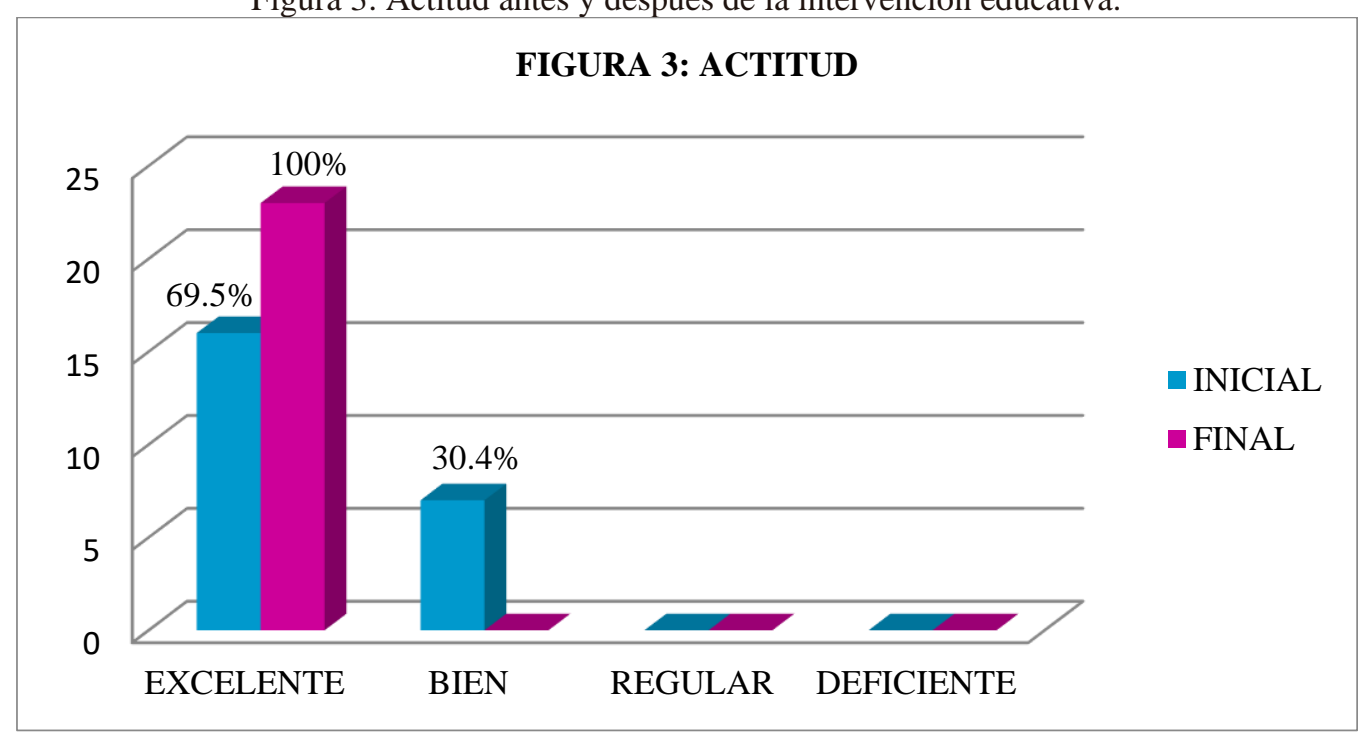

En la figura 4 se muestra que la mayoría de los médicos internos de pregrado culminaron su rotación por medicina familiar con una aptitud excelente.

Figura 4: Aptitud antes y después de la intervención educativa

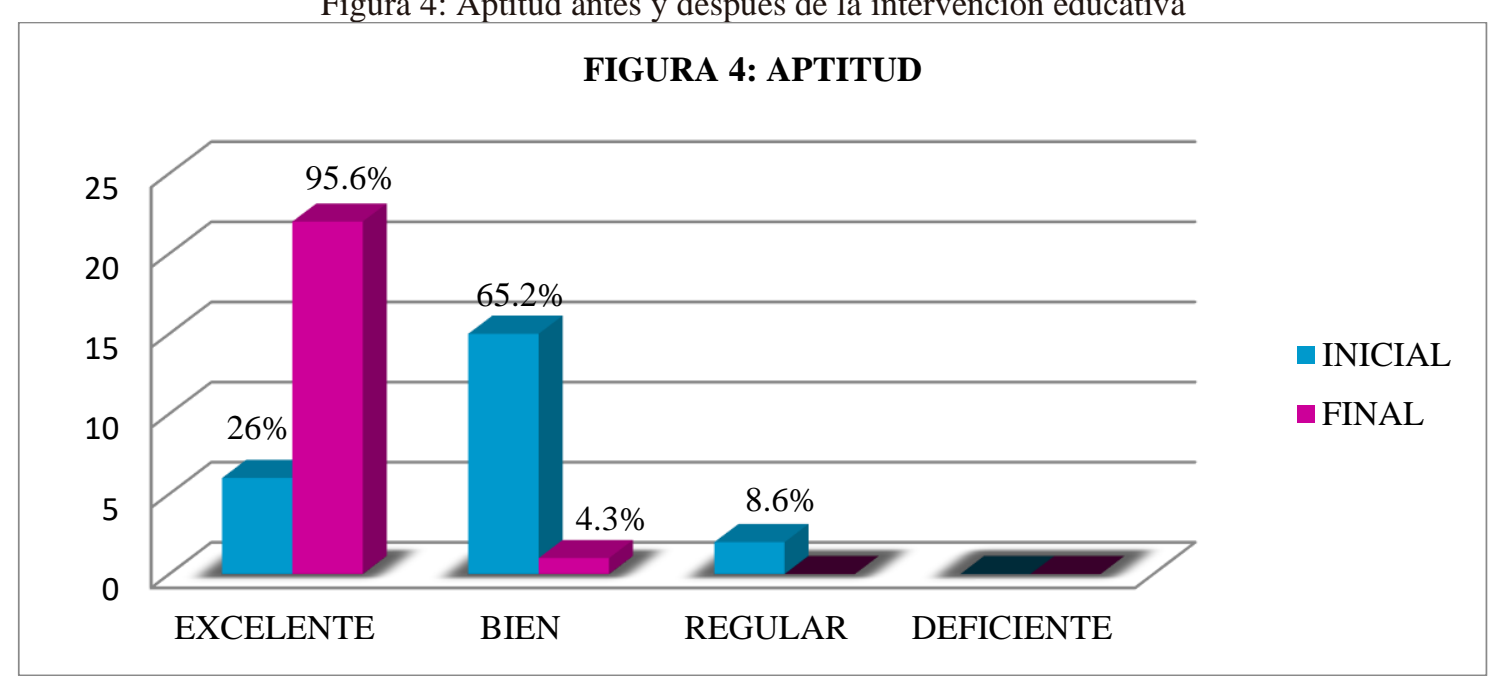

En la figura 5 se observa la evaluación de las competencias profesionales, donde se incluyen los conocimientos, habilidades, actitudes y aptitudes de los médicos internos de pregrado al iniciar y finalizar su rotación por medicina familiar; donde se muestra que el total de los médicos culminaron competentes al egresar de la rotación. 
Figura 5: Competencias profesionales antes y después de la intervención educativa.

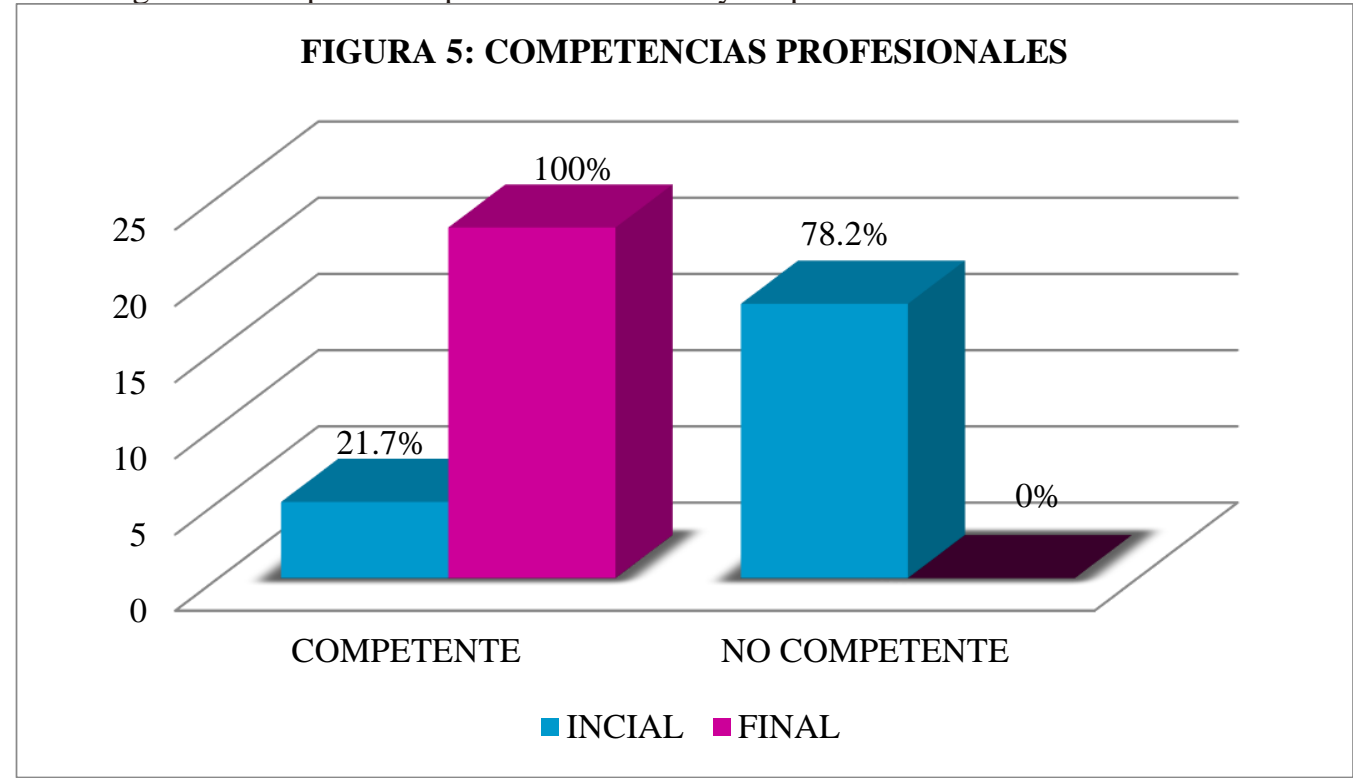

\section{DISCUSIÓN}

Este estudio de investigación reporta el logro de las competencias profesionales de los médicos internos de pregrado en el Instituto Mexicano del Seguro Social al finalizar la rotación por medicina familiar en la UMF 80 de Morelia, Michoacán.

\subsection{EN RELACIÓN CON LA FASE TEÓRICA}

En este estudio se evaluaron a 23 médicos internos de pregrado egresados de la Universidad Michoacana de San Nicolas de Hidalgo (UMSNH) y de la Universidad Autónoma de Guadalajara (UAG) donde la calificación final global que se obtuvo en el área de conocimientos fue de 5.8 en comparación con un estudio que realizó Martínez (2017) en la escuela de medicina más grande del país, UNAM, donde la calificación global fue de 6.2, aplicando la evaluación a un número importante de alumnos, 523 estudiantes de noveno semestre, previo al ingreso del internado médico. Así como Gómez (2019) que realizó un estudio cuantitativo a 80 alumnos del servicio social obteniendo un promedio de 6.6. Con esto podemos notar la similitud de la calificación obtenida en los estudios, demostrando la calidad y similitud de plan académico de estas universidades.

Los hallazgos de este estudio muestran que los alumnos tuvieron un incremento en las seis áreas de conocimiento evaluadas. Donde existió un mayor incremento de conocimientos fue en sobrepeso, infecciones vaginales e infecciones de vías urinarias, en contraste con los hallazgos de Martínez (2017) donde menciona que las áreas de conocimiento con mayor puntuación fueron ginecología, pediatría y propedéutica. 
Se encontró que predominan las habilidades, actitudes y aptitudes sobre los conocimientos en los médicos evaluados, a diferencia del estudio realizado por Bonal (2016) a médicos de familia donde se identificaron conocimientos, actitudes y habilidades resaltando la necesidad de más habilidades y actitudes que de conocimientos, pero esto considerando que los médicos de familia han obtenido más conocimientos durante su trayectoria de la especialidad que los médicos que se encuentran en formación.

\subsection{EN RELACIÓN CON LAS HABILIDADES}

De las seis patologías evaluadas, diabetes mellitus fue donde los médicos internos demostraron su mayor crecimiento en habilidades, colocándose el total de alumnos en categoría de excelente; mostrando su habilidad para evaluar las complicaciones más comunes de la patología y la habilidad de solicitar e interpretar los estudios solicitados; a comparación de el estudio realizado en 2017 por Martínez, donde los puntajes más altos que obtuvieron fue en habilidades de comunicación y los puntajes más bajos fueron obtenidos en la interpretación de exámenes de laboratorio y exploración física; así como las áreas más olvidadas se encontraron las habilidades clínicas.

Al finalizar el estudio, el 100\% de médicos internos fueron hábiles con excelencia en diabetes mellitus, 95\% para infecciones de vías urinarias, 91\% para hipertensión arterial y 86\% para obesidad e infecciones vaginales; similares resultados a los obtenidos en un estudio realizado por Ávila (2016) donde se evaluaron a 17 médicos internos ante 7 escenarios de simuladores donde todos culminaron con $92 \%$ de logro, esto resulta un poco similar ante nuestro estudio excepto en 2 de las 7 estaciones no se logró alcanzar el 90\%; a diferencia del estudio realizado por Sánchez en 2019 donde participaron 184 alumnos que cursaban la carrera de médico cirujano en el cual los resultados mostraron que poco más del 30\% de los alumnos no es hábil en general, hallazgo que hace crítico su desempeño académico y profesional en el futuro.

\subsection{EN RELACIÓN CON LA APTITUD Y ACTITUD}

En este estudio se demostró que el total de los médicos internos egresaron con actitud excelente para poder otorgar una atención digna al paciente, en contraste con lo obtenido en el estudio de Moreno (2020) realizado a 50 médicos del IMSS donde se obtuvo un dominio de actitud solo en el $42.1 \%$ de la población estudiada. Además en nuestro estudio la comunicación, la empatía y el respeto fueron los atributos con valores más altos; similar a los resultados obtenidos en el estudio de Martínez (2017) donde la comunicación, el interrogatorio y la evaluación por el paciente fueron los atributos más altos.

En el presente estudio se muestra que las mujeres presentan niveles más bajos de actitud y empatía a diferencia del estudio de Grau (2017) donde los hombres presentan niveles de empatía más bajos que 
las mujeres, esto también relacionado a que en nuestro estudio el mayor porcentaje de alumnos estudiados fueron mujeres.

En cuanto a la aptitud de los médicos internos, el 95\% se encontraron en excelencia o nivel alto al finalizar su rotación por medicina familiar, un valor más alto que el encontrado en el estudio de Gómez (2019) donde evaluaron a médicos y $60 \%$ se encontraron en nivel medio y solo $7 \%$ en nivel alto.

\subsection{EN RELACIÓN CON LAS COMPETENCIAS PROFESIONALES}

El 100\% de los médicos internos evaluados en este estudio demostraron ser competentes al egresar de su rotación por medicina familiar, incrementando de $21.7 \%$ a $100 \%$ en esta categoría, un notable crecimiento, a diferencia del estudio de Ávila en 2016 donde se evaluaron 17 médicos internos de $7^{\circ}$ año de medicina que obtuvieron $92 \%$ de logro al finalizar los escenarios.

En este estudio se realizó el ECOE para poder evaluar las habilidades coincidiendo con Sureda (2017) que realizó una revisión narrativa de 62 artículos donde se comenta que el ECOE es un instrumento de evaluación apto para la formación universitaria mientras que en el ejercicio profesional se valoran las competencias utilizando la técnica $360^{\circ}$.

Al finalizar este estudio se demuestra que el total de los alumnos fueron competentes en las seis áreas o patologías evaluadas al egresar de su rotación por medicina familiar, comparando lo obtenido con Martínez (2017) donde en su estudio se evaluaron 7 áreas y solo en 5 de ellas es donde se lograron más aciertos.

La limitación más grande del estudio es la proporción de los alumnos que fueron evaluados. Esto se suscitó por el surgimiento de la pandemia por COIVID-19 que limitó los espacios de trabajo y cambios de actividades de los médicos internos dentro de la unidad de medicina familiar.

Otra de las limitantes son el número de las patologías que se implementaron en las estaciones, debido al escaso tiempo con el que se cuenta presencialmente a los alumnos, pero es importante que estudios posteriores se incrementen el número de patologías.

En el estudio solo se aplicaron seis estaciones del ECOE lo cual restringe la cantidad de competencias medidas, y en cuantas más estaciones tenga el ECOE mejor será para obtener conclusiones más sólidas.

\section{CONCLUSIONES}

$\mathrm{Al}$ evaluar las competencias profesionales de los médicos internos de pregrado se concluye que son competentes al egresar de su rotación de medicina familiar, encontrando un impacto favorable con la intervención educativa que se realizó durante el estudio. 
Se encontró un cambio en los conocimientos de los médicos internos de pregrado, principalmente en las patologías de sobrepeso y obesidad, infecciones vaginales e infecciones de vías urinarias.

Los alumnos lograron desarrollar habilidades en búsqueda y prevención de complicaciones de diabetes mellitus, hipertensión arterial, sobrepeso, en el control adecuado de la embarazada y en detección de infecciones de vías urinarias y vaginales.

La actitud y aptitud de los médicos internos de pregrado presenta mejoría notable posterior a la evaluación y retroalimentación otorgada. 


\section{REFERENCIAS BIBLIOGRÁFICAS}

Ávila, R., Mahana, P., Rivera, C., \& Mc Coll, P. (2016). Simulación clínica como método de formación de competencias en estudiantes de medicina. Revista Educación en Ciencias de la Salud, 13(1), 11-14. Recuperado de: http://www2.udec.cl/ofem/recs/anteriores/vol1312016/artinv13116a.pdf

Beneitone, P., González, J. y Wagenaar, R. (2014). Meta-perfiles y perfiles. Una nueva aproximación para las titulaciones en América Latina. España: Proyecto Tuning America. Recuperado de: http://www.deusto-publicaciones.es/deusto/pdfs/tuning/tuning71.pdf

Bonal-Ruiz, R., Marzán-Delis, M. y Garbey-Acosta, A. (2017). Identificación de competencias en promoción de salud en médicos de familia de Cuba Estudio cualitativo. Atención Familiar, 24(1), 32-39. Recuperado de: https://www.medigraphic.com/pdfs/atefam/af-2017/af171h.pdf

Cresp, M., Espinoza, V., Machuca, C., García, R., Carter, A. y Sanhueza, S. (2018). Evaluación Clínica Objetiva Estructurada (ECOE) como proceso de innovación y seguimiento del perfil profesional de la carrera de Nutrición y Dietética de la Universidad Católica de Temuco, Chile. Dilemas Contemporáneos: Educación, Política y Valores, 2(28), 1-1. Recuperado de: https://www.dilemascontemporaneoseducacionpoliticayvalores.com/index.php/dilemas/article/view/425 1466

Gómez, V. y Rosales, S. (2019). Evaluación de la aptitud clínica de los médicos pasantes en servicio social. Investigación en educación médica, 8(31), 55-63. Recuperado de: http://www.scielo.org.mx/scielo.php?pid=S2007-50572019000300055\&script=sci_abstract\&tlng=pt

Grau, A., Torán, P., Zamora, A., Quesada, M., Carrion, C., Vilert, E. y Cordon, F. (2017). Evaluación de la empatía en estudiantes de Medicina. Educación médica, 18(2), 114-120. Recuperado de: https://www.sciencedirect.com/science/article/pii/S1575181316300419

Martínez-González, A., Lifshitz-Guinzberg, A., González-Quintanilla, E., Monterrosas-Rojas, A., FloresHernández, F., Gatica-Lara, F. y Sánchez-Mendiola, M. (2017). Nivel de competencia docente en el Internado Médico de Pregrado de la Facultad de Medicina de la UNAM. Revista Médica del Instituto Mexicano del Seguro Social, 55(6), 778-787. Recuperado de: https://www.redalyc.org/jatsRepo/4577/457753402025/457753402025.pdf

Martínez-González, A. y Soto-Estrada, G. (2018). Examen Clínico Objetivo Estructurado. ¿El reto a vencer para ejercer la medicina? Revista Digital Universitaria, 9 (6), 1-12. Recuperado de: http://www.revista.unam.mx/wp-content/uploads/v19_n6_a12_Examen-Cl\%C3\%ADnico-ObjetivoEstructurado.-El-reto-a-vencer-para-ejercer-la-medicina.pdf

Moreno, O., Carillo, L., González, B., Salas, R. y Olvera, V. (2020). Conocimientos, actitudes y prácticas de los profesionales de la salud ante el VIH/SIDA en el primer nivel de atención. South Florida Journal of Development, 1(4) 221-229. Recuperado de: https://southfloridapublishing.com/ojs/index.php/jdev/article/view/58/70

Nieto-Gutierrez, W., Taype-Rondan, A., Bastidas, F., Casiano-Celestino, R. e Inga-Berrospi, F. (2016). Percepción de médicos recién egresados sobre el internado médico en Lima, Perú 2014. Acta Médica Peruana, 33(2), 105-110. Recuperado de: http://www.scielo.org.pe/scielo.php?script=sci_arttext\&pid=S1728-

$59172016000200003 \& \operatorname{lng}=\mathrm{es} \& \ln g=\mathrm{es}$ 
Rodríguez, R., Hernández, N. y Díaz, F. (2007). Docencia universitaria: cómo planificar asignaturas para el aprendizaje de competencias: primeros pasos para el diseño de un plan docente. España: Oviedo. Recuperado de: https://www.worldcat.org/title/docencia-universitaria-como-planificar-asignaturas-parael-aprendizaje-de-competencias-primeros-pasos-para-el-diseno-de-un-plan-docente/oclc/804201107

Salas-Flores, R. y González-Perez, B. (2016). Implementación del día académico en médicos internos de pregrado. Revista Médica del Instituto Mexicano del Seguro Social, 54 (3). 386-390. Recuperado de: https://www.redalyc.org/pdf/4577/457745710018.pdf

Sánchez, A., Flores, R., Urbina, B. y Lara, N. (2008). Expectativas y realidades del internado médico de pregrado. Un estudio cualitativo. Investigación en salud, 10 (1), 14-21. Recuperado de: https://www.redalyc.org/pdf/142/14219995004.pdf

Sánchez, A., Osornio, L. y Ríos, M. (2019). Habilidades sociales básicas y su relación con la ansiedad y las estrategias de afrontamiento en estudiantes de medicina. Revista Electrónica de Psicología Iztacala, 22 (2), 834-856. Recuperado de: http://www.revistas.unam.mx/index.php/repi/article/view/70134

Soler, M. (2004). Reflexiones acerca del término de competencias en la actividad docente. Educación Médica Superior, 18 (1). Recuperado de: http://scielo.sld.cu/scielo.php?pid=S0864$21412004000100005 \&$ script=sci_arttext\&tlng=en

Sureda-Demeulemeester, E., Ramis-Palmer, C. y Sesé-Abad, A. (2017). La evaluación de competencias en Medicina. Revista Clínica Española,217(9), 534-542. Recuperado de: https://www.sciencedirect.com/science/article/abs/pii/S0014256517301479

Tapia, R., Núñez, R., Salas R. y Rodríguez A. (2007). El internado médico de pregrado y las competencias clínicas. México en el contexto latinoamericano. Educación Media Superior, 21 (4). Recuperado de: http://scielo.sld.cu/scielo.php?pid=S0864-21412007000400005\&script=sci_arttext\&tlng=en

Ticse, R. (2017). El Examen Clínico Objetivo Estructurado (ECOE) en la evaluación de competencias de comunicación y profesionalismo en los programas de especialización en Medicina. Revista Medica Herediana, 28 (3), 192-199. Recuperado de: http://www.scielo.org.pe/scielo.php?script=sci_arttext\&pid=S1018-130X2017000300010

Tobón, S., Pimienta, J. y García, J. (2010). Secuencias Didácticas: Aprendizaje y Evaluación de Competencias. México: PEARSON. Recuperado de: https://www.researchgate.net/profile/Sergio_Tobon4/publication/287206904_Secuencias_didacticas_apr endizaje_y_evaluacion_de_competencias/links/567387b708ae04d9b099dbb1.pdf

Trejo-Mejía, J., Blee-Sánchez, G. y Peña-Balderas, J. (2013). Elaboración de estaciones para el Examen Clínico Objetivo Estructurado (ECOE). Investigación en Educación Médica, 3 (9), 56-59. Recuperado de: https://www.elsevier.es/en-revista-investigacioneducacion-medica-343-articulo-elaboracion-estacionesel-examen-clinico-S2007505714727255

Trejo, J., Martínez, A., Méndez, I., Morales, S., Ruiz, L. y Sánchez, M. (2014). Evaluación de la competencia clínica con el examen clínico objetivo estructurado en el internado médico de la Universidad Nacional Autónoma de México. Gaceta Médica de México, 150(1), 8-17. Recuperado de: https://www.medigraphic.com/pdfs/gaceta/gm-2014/gm141b.pdf 
Vidal, M., Salas, R., Fernández, B. y García, A. (2016), Educación basada en competencias. Revista cubana de educación media superior, 30 (1). 1-13. Recuperado de: https://www.medigraphic.com/pdfs/educacion/cem-2016/cem161r.pdf 